() 2016, Elsevier. Licensed under the Creative Commons Attribution-NonCommercial-NoDerivatives 4.0 International http://creativecommons.org/licenses/by-nc-nd/4.0/

Neural activity during object perception in schizophrenia patients is associated with illness duration and affective symptoms

Christian Stephan-Otto, PhD ${ }^{\mathrm{a}, \mathrm{b}}$, Sara Siddi, MSc ${ }^{\mathrm{a}, \mathrm{c}, \mathrm{d}}$, Jorge Cuevas Esteban, MD, PhD ${ }^{\mathrm{a}}$, Carl Senior, $\mathrm{PhD}^{\mathrm{e}}$, Roberto García-Álvarez, PhD $^{\mathrm{f}}$, Maria Rosa Cambra-Martí, MD ${ }^{\mathrm{g}}$, Judith Usall, $\mathbf{M D}$, PhD ${ }^{\mathrm{a}}$, Gildas Brébion, $\mathbf{P h D}^{\mathbf{a}^{*}}$

${ }^{\text {a }}$ Parc Sanitari Sant Joan de Déu, Sant Boi de Llobregat, Spain, and CIBERSAM

${ }^{\mathrm{b}}$ Fundació Sant Joan de Déu, Barcelona, Spain

${ }^{c}$ Section of Clinical Psychology, Department of Education, Psychology, and Philosophy, University of Cagliari, Italy

${ }^{\mathrm{d}}$ Facultat de Medicina, Universitat de Barcelona, Spain.

${ }^{\mathrm{e}}$ School of Life \& Health Sciences, Aston University, Birmingham, UK

${ }^{\mathrm{f}}$ Research and Collaborations, General Electric Healthcare, Buc, France

${ }^{\mathrm{g}}$ Diagnostic Imaging Unit, Parc Sanitari Sant Joan de Déu, Sant Boi de Llobregat, Spain

* Corresponding author: Gildas Brébion, Ph.D.

Unitat de Recerca, Parc Sanitari Sant Joan de Déu, 08830 Barcelona, Spain

Tel. (34) 936406350 ext. 12544. E-mail: gildas.brebion@pssjd.org 


\begin{abstract}
Background: Abnormalities in visual processes have been observed in schizophrenia patients and have been associated with alteration of the lateral occipital complex and visual cortex. However, the relationship of these abnormalities with clinical symptomatology is largely unknown. Methods: We investigated the brain activity associated with object perception in schizophrenia. Pictures of common objects were presented to 26 healthy participants (age $=36.9 ; 11$ females) and 20 schizophrenia patients (age $=39.9 ; 8$ females) in an fMRI study. Results: In the healthy sample the presentation of pictures yielded significant activation $(\mathrm{p}<.001)$ of the bilateral fusiform gyrus, bilateral lingual gyrus, and bilateral middle occipital gyrus. In patients, the bilateral fusiform gyrus and bilateral lingual gyrus were significantly activated ( $\mathrm{p}$ $<.001$ ), but not so the middle occipital gyrus. However, significant bilateral activation of the middle occipital gyrus $(\mathrm{p}<.05)$ was revealed when illness duration was controlled for. Depression was significantly associated with increased activation, and anxiety with decreased activation, of the right middle occipital gyrus and several other brain areas in the patient group. No association with positive or negative symptoms was revealed. Conclusions: Illness duration accounts for the weak activation of the middle occipital gyrus in patients during picture presentation. Affective symptoms, but not positive or negative symptoms, influence the activation of the right middle occipital gyrus and other brain areas.
\end{abstract}

Keywords: schizophrenia, fMRI, object perception, middle occipital gyrus, affective symptoms 


\section{Neural activity during object perception in schizophrenia patients is associated with illness duration and affective symptoms}

\section{INTRODUCTION}

Visual perception abnormalities have been observed in patients with schizophrenia and, notably, have been found to be associated with under-activation and impaired coupling of the lateral occipital complex (Green et al., 2009; Harvey et al., 2011). This area is involved in object perception and recognition (Cichy et al., 2012; Grill-Spector et al., 2001; Macevoy, 2013). Alterations were also observed in various areas of the visual cortex (Schultz et al., 2013; Sehatpour et al., 2010; Silverstein et al., 2009). At the clinical level, visual abnormalities in patients with schizophrenia have been related to negative symptomatology (Bedwell et al., 2011; Green et al., 2011; Lee et al., 2010; Perez et al., 2012; Slaghuis and Curran, 1999). A study with evoked potentials recently reported that negative symptoms were related to perceptual processing dysfunction in these patients (González-Hernández et al., 2014). Whether negative symptoms are related to cerebral under-activation during visual perception has not been clearly established.

Although affective symptoms are not routinely assessed in patients with schizophrenia, several groups have reported them to play a significant role in memory (Lysaker et al., 2000; Möser et al., 2006; Sanfilipo et al., 2002) and attention (Möser et al., 2006) in this population. Our previous work suggested that depression and/or anxiety affected verbal (Brébion et al., 2013, 2009, 2001) as well as visual (Brébion et al., 2015) memory, while potential associations between memory and negative symptoms were mostly an artifact of the overlap between affective and negative symptoms. Depression and anxiety, rather than negative symptoms, might similarly 
have some effect on visual perception. Indeed, basic visual perceptual deficits have been reported in depressed patients (Clark et al., 2014).

The brain areas involved in visual perception have been clearly established in healthy participants. A recent meta-analysis of functional magnetic resonance imaging (fMRI) studies revealed that viewing of pictures of art was associated with activation of vision-related as well as emotion-related brain areas (Vartanian and Skov, 2014). The identified visual brain areas included the lingual gyrus, the middle occipital gyrus, and the fusiform gyrus. The precuneus, allegedly involved in visuo-spatial exploration of visual stimuli, was also identified, as were the inferior temporal gyrus, involved in visual representation of forms and colours, the parahippocampal gyrus, involved in the perception and recognition of scenes, and the superior temporal gyrus, which might be related to the semantic processing of objects within the pictures. The additional activation of emotional areas was attributed to the aesthetic component of the paintings. These areas included the putamen, presumably associated with the anticipation of rewards, as well as the anterior insula. A recent review showed the critical role of the insula in emotion (Gasquoine, 2014).

In this study we attempted to identify some of the brain structures involved in impaired visual perception in schizophrenia, and the clinical factors that may be related to abnormal activation of these structures. The brain areas associated with the viewing of pictures of common objects were investigated in healthy participants and patients with schizophrenia. It was expected that, in healthy individuals, visual, but not emotional, brain areas would be activated. Patients were expected to demonstrate under-activation of the same brain areas. Further, we hypothesized that these brain 
abnormalities were associated with negative, but not positive, symptomatology. We sought to determine whether affective symptoms (depression and anxiety), allegedly related to negative symptoms, presented similar associations with brain activation as they did, and might even account for the associations with negative symptoms.

Potential associations between brain abnormalities and illness duration were explored.

\section{METHOD}

\subsection{Participants}

Forty-eight participants were tested, but data from the functional series were disregarded for one healthy participant and one patient due to machine errors. After removing these two participants, the following participant samples were obtained: Twenty-six healthy participants (11 females) with normal or corrected-to-normal vision were recruited from the general population by means of announcements displayed in the public access areas of the hospital campus: age: $\mathrm{m}=36.9$, $\mathrm{sd}=9.3$; education level: $\mathrm{m}=$ 6.1 ${ }^{1}$; estimated verbal IQ: $m=104^{2}$. The inclusion criteria were age between 18 and 60 years, and fluency in Spanish. The exclusion criteria were neurological or mental disease, intellectual disability, head injury, alcohol or drug abuse in the past six months, and current severe physical disease, as well as the standard exclusion criteria for participation in fMRI procedures, namely claustrophobia, and metallic implants including fitted pacemaker or cochlea implants.

\footnotetext{
${ }^{1}$ The scale used was: 1 = no studies; 2 = uncompleted primary studies; 3 = completed primary studies; 4 = high school uncompleted; $5=$ high school completed; $6=$ uncompleted undergraduate studies; $7=$ bachelor's or master's degree; 8 = doctorate

${ }^{2}$ Verbal IQ was assessed by means of the Acentuación de Palabras test (TAP). Equivalence with standard verbal IQ was estimated according to norms $(I Q=104$ for $T A P=24.3$, and IQ $=98$ for $T A P=$ 21.1) (Gomar et al., 2011).
} 
Twenty patients with schizophrenia (DSM-IV criteria, 8 females) were recruited from the Parc Sanitari Sant Joan de Déu network of mental health services in Barcelona, Spain: age: $\mathrm{m}=39.9, \mathrm{sd}=11.5$; education level: $\mathrm{m}=4.8^{1}$; verbal IQ: $\mathrm{m}=98^{2}$. Illness duration $^{3}: \mathrm{m}=12.8, \mathrm{sd}=9.9$. The inclusion criteria were the same as for healthy participants with the additional criterion of being able to provide informed consent. The exclusion criteria were also the same except that the exclusion of mental disease only applied to organic mental disorders and dementia.

The two groups were equivalent for age and sex distribution. However, the healthy controls presented significantly higher educational level $(\mathrm{t}(44)=2.9, \mathrm{p}<.01)$ and verbal IQ $(\mathrm{t}(44)=2.1, \mathrm{p}<.05)$ than did the patients. All of the procedures were approved by the Parc Sanitari Sant Joan de Déu ethics committee, and all participants provided informed consent before taking part in the study.

\subsection{Clinical rating scales}

Positive and negative symptoms were assessed in patients using the Spanish version of the Scale for the Assessment of Positive Symptoms (SAPS) and the Scale for the Assessment of Negative Symptoms (SANS) (Peralta and Cuesta, 1999). The scores tallied for positive symptoms were hallucinations, delusions, thought disorganisation, and total SAPS scores. The scores tallied for negative symptoms were flat affect, alogia, avolition, anhedonia, attention disorder, and total SANS scores. Affective symptoms were assessed by means of the Calgary Depression Scale and the Hamilton Anxiety Rating Scale. Clinical assessment was conducted shortly after the completion of the task by a trained clinical psychologist who was blind to the experimental

\footnotetext{
${ }^{3}$ Illness duration was calculated as the number of years elapsed since the first psychiatric hospitalization.
} 
hypotheses. The main scores obtained were: SAPS: $\mathrm{m}=14.9, \mathrm{sd}=16.6$, range $[0-51]$; SANS: $\mathrm{m}=4.7, \mathrm{sd}=6.0$, range [0-18]; Calgary Depression Scale: $\mathrm{m}=2.7, \mathrm{sd}=3.8$, range [0-14]; Hamilton Anxiety Rating Scale: $\mathrm{m}=6.2$, $\mathrm{sd}=4.4$, range [0-17].

\subsection{Material and procedure}

All procedures reported here form part of a task designed to examine realitymonitoring deficits in schizophrenia. Participants were first presented with a mixture of target words and pictures (encoding phase). Then, in the recall phase, they were presented with the labels of the target items and required to remember whether a picture of the item had been presented at encoding. This current report focuses on the perceptual aspects of the task, and therefore only the encoding phase (presentation of words and pictures) will be described (see Figure 1).

Ninety items were selected, including 72 common objects (e.g., saw, apron, envelope) and 18 vegetables (e.g., carrot, cauliflower, onion). Participants were presented with either the verbal label of the item alone or the picture of the item along with its verbal label. Half of the items were presented as word only and the other half were presented as word/picture pairs. Two versions of the stimuli were prepared; the 45 stimuli that were presented as words in one version were presented as word/picture pairs in the other. The use of each version was counterbalanced among subjects.

All material was presented to participants throughout via Presentation (http://www.neurobs.com/). The items were presented one by one in pseudo-random order with each slide (word or word/picture pair) being presented for 3.5 seconds, separated by fixation-crosses. To control for visual habituation of the presented material all stimuli were presented in jittered fashion with the stimulus onset 
asynchrony varied across trials from 2 to 5.5 seconds according to an exponential distribution with mean $=3.18$ seconds. Participants were asked to indicate whether the presented items were vegetables or not, and to give their response by pressing one of two buttons ('vegetable' or 'other'). This procedure was used to ensure effective processing of the presented items. The number of errors was negligible.

\section{4. fMRI data acquisition}

Functional MRI data were acquired with a 1.5T General Electric Signa HDe scanner at Parc Sanitari Sant Joan de Déu. A T2*-weighted functional echoplanar imaging sequence depicting BOLD contrast was obtained using a quadrature head coil. In total 270 volumes were collected with slices parallel to the AC-PC plane, resulting in an axial-oblique orientation. The following scanning parameters were used: 26 slices, $4 \mathrm{~mm}$ thickness, $1 \mathrm{~mm}$ gap, TR $=2000 \mathrm{~ms}, \mathrm{TE}=40 \mathrm{~ms}, 24 \mathrm{~cm} \mathrm{FOV}, 64 \times 64$ acquisition matrix, flip angle $=90^{\circ}$. The first four volumes in each run were discarded to allow for magnetic saturation effects. Visual stimuli were presented on a rearprojection screen and viewed through a mirror mounted on the head coil, and all responses were collected with an MR-compatible response box (fORP, Current Designs, Inc., USA; www.curdes.com).

\section{5. fMRI data preprocessing}

Imaging data were analyzed using SPM8 (Wellcome Department of Imaging Neuroscience, London; www.fil.ion.ucl.ac.uk/spm) running under MATLAB (Release 2009a, The MathWorks, Inc., Natick, Massachusetts). All of the volumes from each participant were spatially realigned to the first image in each series, in order to correct for small head movements and to generate a mean image for the functional series. 
Motion parameters were examined for each subject to ensure no movements larger than the voxel size were present (no runs were discarded). The resulting series were transformed into SRI24 space (Rohlfing et al., 2010), with a standard EPI template as deformation target, and then spatially smoothed using a Gaussian kernel of $8 \mathrm{~mm}$ fullwidth-at-half-maximum.

\section{6. fMRI data analysis}

Preprocessed fMRI data were then analyzed with an event-related model, using SPM8. In order to assess random effects at the individual level, the activity associated with the experimental conditions was modeled by a hemodynamic response function (HRF) and its time derivative. Displacement and rotation motion parameters were included as confounds in the individual model. A $200 \mathrm{~Hz}$ high-pass filter cutoff was used to remove low frequency noise, together with an AR(1) model to correct for temporal autocorrelation.

Two event types were determined by the composition of the stimuli: Word events (for word-only stimuli) and Picture events (for word/picture pairs). A linear contrast Pictures $>$ Words was constructed to test the activation linked to picture perception. This contrast was entered into a second level analysis in which subjects were treated as a random effect.

The corresponding statistical parametric maps were generated with SPM8, using an uncorrected voxel-level threshold defined by $\mathrm{p}<.001$ and a cluster extent threshold defined by a family-wise-error (FWE) corrected $\mathrm{p}<.05$. In the event that the resulting activation clusters encompassed several brain structures, a more restrictive voxel-wise threshold, FWE-corrected $\mathrm{p}<.05$ at voxel level, was applied to better identify local activation maxima in each brain structure. The reported activation peaks were labelled 
according to the SRI24/TZO cortical parcellation map (Rohlfing et al., 2010) based on the template described by Tzourio-Mazoyer et al. (2002).

\subsection{Plan of analysis}

The contrast Pictures $>$ Words was studied in the healthy and patient groups to reveal the brain activation associated with the presentation of pictures. In each group, this contrast was first conducted with sex, verbal IQ, education level, and age included in the analysis as covariates so as to explore the potentially confounding effect of these sociodemographic factors. Then, the contrast Pictures $>$ Words was re-conducted after controlling for identified confounders, if any.

Subsequently, regression analyses were conducted in the patient group by means of SPSS (version 19.0. Armonk, NY: IBM Corp.) to study the associations between clinical symptoms and the observed activations of vision-related brain regions. The dependent variables were the local activation maxima corresponding to the different brain structures identified within each activation cluster. Two models were tested, one including SANS, depression and anxiety scores, and the second including SAPS, depression, and anxiety scores, as independent variables. Finally, we explored potential brain activations associated with each negative, positive, and affective symptom score, as well as with illness duration, during the presentation of pictures. This was achieved by entering each variable alternately in the analysis when conducting the contrast Pictures $>$ Words. 


\section{RESULTS}

\subsection{Brain activation in healthy participants}

In the 26 healthy participants, the contrast Pictures $>$ Words with the four sociodemographic variables entered as covariates yielded a significant effect of verbal IQ, in the sense that higher verbal IQ was associated with increased activation in the right precentral gyrus (cluster-level, FWE corrected $\mathrm{p}<.05$, after a global $\mathrm{p}_{\text {peak }}<.001$, maximum activation peak at $33,-37,56$ in SRI24 coordinates). No significant effects were found for sex, education level, or age. When the contrast Pictures $>$ Words was computed after covariating only verbal IQ, significant activation was observed in the bilateral fusiform gyrus, the bilateral lingual gyrus, and the bilateral middle occipital gyrus (see Table 1$)^{4}$.

\subsection{Brain activation in schizophrenia patients}

In the 20 schizophrenia patients, no significant effect of verbal IQ on brain activation was revealed, contrary to what was observed in the healthy participants. Sex, education level and age did not yield any significant activation either. The contrast Pictures $>$ Words computed without covariating any factor yielded activation in the same visual areas as in the healthy control participants (see Table 1). However, underactivation of the middle occipital gyrus was observed: the left middle occipital gyrus

\footnotetext{
${ }^{4}$ Considering the significant effect of verbal IQ and the fact that the healthy control group presented higher verbal IQ than the patient group, we recomputed the contrast Pictures $>$ Words, covariating verbal IQ, after removing the three healthy participants with the highest verbal IQ. This subgroup of healthy participants was equivalent to the patient group with regard to verbal IQ $(p>.10)$. When the analysis was restricted to this healthy subgroup, significant activations were observed in the bilateral fusiform gyrus, the bilateral lingual gyrus, and the bilateral middle occipital gyrus, equivalent to what was observed in the whole healthy group, and with the same level of statistical significance.
} 
was not significantly activated after FWE correction, while the right one was significantly activated only at peak level (FWE-corrected).

When the 20 patients were compared to the 26 healthy participants for the contrast Pictures > Words after controlling for verbal IQ, no significant differential activation emerged in the healthy group, contrary to our expectations. This suggests that the patients were not abnormal in their brain activation.

\subsection{Associations between brain activation and clinical measures in patients}

\subsubsection{Intercorrelations among the clinical measures}

Correlational analyses indicated that the SAPS score was unrelated to SANS, depression, or anxiety score. In contrast, the SANS score was significantly associated with both depression and anxiety scores, as expected $(\mathrm{r}=.63, \mathrm{p}<.005$, for both correlations). Anxiety and depression scores were positively and significantly intercorrelated $(\mathrm{r}=.58, \mathrm{p}<.01)$. Illness duration was not associated with any of these symptom scores.

\subsubsection{Vision-related brain area activations and clinical symptoms}

Regression analyses were conducted on the four local activation maxima reported in Table 1. The first model including SANS, depression, and anxiety scores as independent variables did not reveal any significant association for the bilateral lingual gyrus, the left fusiform gyrus, or the right fusiform gyrus. Activation in the right middle occipital gyrus was not inversely associated with SANS score, contrary to our expectations $(\beta=.27, p=.33)$. However, it was positively associated with depression $(\beta=.59, \mathrm{p}<.05)$ and negatively associated with anxiety $(\beta=-.72, \mathrm{p}<.015)$. It should be 
noted that a trend toward a positive association with the SANS score was observed when depression was removed from the predictors $(\beta=.53, \mathrm{p}<.10)$.

The second model with SAPS, depression, and anxiety scores as independent variables yielded a similar pattern of results. No significant association with brain activation appeared for the bilateral lingual gyrus or the left or right fusiform gyrus. The activation in the right middle occipital gyrus was positively associated with depression $(\beta=.66, p<.025)$ and negatively associated with anxiety $(\beta=-.60, p<.05)$, while no association with SAPS score emerged $(\beta=-.11, \mathrm{p}=.61)$.

\subsubsection{Brain activations associated with clinical symptoms and illness duration}

No significant activation was observed for any negative symptom, contrary to our expectations. The positive symptoms did not yield any significant activation either. With regard to the affective symptoms, depression and anxiety scores were entered together in the analyses to control for each other's effect, given their above-mentioned opposite association with brain activation. Depression was found to be associated with increased activation in the bilateral calcarine gyrus, right precuneus, and left supramarginal gyrus. Anxiety was found, in contrast, to be associated with decreased activation in the left supra marginal gyrus. It was also associated with decreased activation in the left postcentral gyrus (see Table 2 and Figure 2).

Illness duration was associated with decreased activation in the right angular gyrus, the left cerebellum, and the bilateral middle occipital gyrus (see Table 3). In view of the significant association between illness duration and poor activation of the middle occipital gyrus, we re-examined the contrast Pictures $>$ Words in patients while covariating illness duration. Significant activations in the right and left middle occipital 
gyrus were then revealed (see Table 1), suggesting that illness duration accounted for the previously-mentioned lack of any significant activation of this area in patients.

\section{DISCUSSION}

The viewing of pictures representing common objects activated a network of vision-related brain areas in our sample of healthy participants. This included the bilateral fusiform gyrus, the bilateral lingual gyrus, and the bilateral middle occipital gyrus. These visual brain areas are similar to those identified by Vartanian and Skov (2014) during the viewing of art paintings. However, other brain areas identified by these authors and allegedly involved in visual processes, namely the precuneus, the temporal gyrus, and the parahippocampal gyrus, were not activated by our healthy participants. These regions might be activated only when viewing sophisticated stimuli that involve some visual exploration or integration. As expected, still other brain regions related to emotional and reward systems, and activated by art pictures in the meta-analysis of Vartanian and Skov (2014), were not activated by the presentation of our basic visual stimuli.

Patients with schizophrenia demonstrated activation in the same visual brain areas as did the healthy participants during the viewing of pictures, and group comparison did not reveal any significant brain abnormality in patients. Nonetheless, the data suggest under-activation of the middle occipital gyrus since the patients did not significantly activate the left middle occipital gyrus, while the right middle occipital gyrus was significantly activated at peak level but only tended to be activated at cluster level. The absence of a significant activity difference in this brain region relative to the healthy sample might stem from lack of statistical power. 
Regression analyses including positive or negative symptoms along with affective symptoms showed that negative symptoms were not related to any of these brain activations. In contrast, affective symptoms were found to have significant and opposite effects on the activation observed in the right middle occipital gyrus. Depression was associated with increased activation in this area, while anxiety was associated with decreased activation. It is worth noting that a trend-level association between negative symptoms and increased activation of the right middle occipital gyrus could be observed when depression was removed from the predictors -that is, when the regression analysis included only negative symptoms and anxiety. However, this trend no longer existed when depression was entered in the model. Thus, depression appears to be the genuine predictor of increased brain activation in this area, while the trend association with negative symptoms seems to be a mere artifact of the overlap between negative and depressive symptomatology. This pattern of associations corroborates and extends our previous finding of a role of affective, rather than negative, symptoms in memory performance, observed in several schizophrenia samples (Brébion et al., 2015, 2013, 2009, 2001). Previously reported associations between impaired visual abilities and negative symptoms might similarly have been influenced by depressive symptoms.

In our data, depression was also found to be associated with increased activation in another visual region, the calcarine gyrus, and with increased activation in the right precuneus and the left supramarginal gyrus. The precuneus has been found to be activated during the viewing of paintings in healthy individuals (Vartanian and Skov, 2014). In addition, it may be part of a functional network associated with depression, as suggested by a study with first-episode depression patients (Peng et al., 2015) and a 
study of non-clinical depressive symptoms (Wei et al., 2015). With respect to the supramarginal gyrus, resting state studies provide evidence that hyperactivation of this brain region is involved in depression (Wei et al., 2015; Yin et al., 2015). Meanwhile, anxiety in our data was found to be associated with decreased activation in the left supramarginal gyrus and left postcentral gyrus. These findings are compatible with a recent study that revealed decreased gray matter volume of supramarginal and postcentral gyrus in patients with generalized anxiety disorders (Makovac et al., 2016). Further, anxiety has been associated with decreased activation (Ball et al., 2014; Brown et al., 2015) and functional connectivity (Modi et al., 2015) of the supramarginal gyrus in individuals with high levels of anxiety. Our data suggest that these brain abnormalities associated with affective symptoms are also present in patients with schizophrenia. The observed opposite associations of anxiety and depression with brain activity indicate that associations with each of these symptoms are more likely to emerge when the other symptom is controlled. This might be particularly relevant for the supramarginal gyrus, which appears to be consistently hyperactivated in depressive states and hypoactivated in anxious states.

Positive associations of brain activity with depression could mean that greater effort has to be made by depressed people even in basic perceptual processes, resulting in increased cerebral activation. In contrast, considering the putative role of anxiety in the detection of potential dangers, decreased brain activation along with anxiety could point to enhanced perceptual alertness in anxious individuals. In the same vein, an auditory study in healthy individuals revealed that anxiety increased the perception of an auditory warning signal while depression reduced it (Riskind et al., 2014). 
Anxiety and depression are prevalent in patients with schizophrenia and have been found to be associated with psychotic symptoms as well as with functional and social outcome (Hartley et al., 2013; Lysaker and Salyers, 2007; Schennach-Wolff et al., 2011; Wetherell et al., 2003). At the cognitive level, several groups have reported that anxiety and depression play a significant role in memory and attention. The present results reveal that these affective symptoms also influence basic visual perception, while positive and negative symptoms do not. Future studies of visual perception should employ more complex pictures capable of inducing emotional reactions, rather than the neutral objects used in our study. Schizophrenia patients could be expected to demonstrate under-activation of the emotional brain areas -notably the insula- allegedly activated by the healthy participants. The extent to which such brain under-activation might be accounted for by anxiety and/or depression rather than by certain negative symptoms such as blunted affect or anhedonia could be determined. Future studies of schizophrenia could also examine the impact of affective symptoms on perceptual processes in other modalities. Lastly, it would be worthwhile to investigate whether similar opposite effects of anxiety and depression on perception can be observed in other clinical populations as well as in the general population.

Another clinical factor that appears to have an impact on brain activity in patients is illness duration. Indeed, the number of years elapsed since the onset of the disease was significantly associated with decreased activation in three brain areas, namely the right angular gyrus, the left cerebellum, and the bilateral middle occipital gyrus. Further, as stated above, the only brain region that showed underactivation in patients was the middle occipital gyrus. Weak activation of the middle occipital gyrus could not be accounted for by negative symptoms since, if anything, negative symptoms 
were associated with increased activation in this area. However, it was accounted for by illness duration. When this factor was controlled for, significant cluster level activation in the left and right middle occipital gyrus was observed in patients. Illness duration might similarly contribute to the under-activation or alteration of the lateral occipital complex and visual cortex reported in this population (Green et al., 2009; Schultz et al., 2013; Sehatpour et al., 2010; Silverstein et al., 2009). Our findings highlight the importance of considering the number of years suffering the disease as a potential cause of decreased brain activation in schizophrenia.

In summary, our results indicate that the bilateral lingual gyrus and bilateral fusiform gyrus were significantly activated in patients during the viewing of pictures of objects. In contrast, the bilateral middle occipital gyrus was under-activated proportionally to the duration of the disease. Activation of the right middle occipital gyrus and several other brain regions was associated with higher levels of depression and/or lower levels of anxiety. The scope of our conclusions is limited by the small number of participants in each group, as is generally the case in brain imaging studies. Apart from the inherent lack of statistical power, this small sample might not be representative of the schizophrenia population. Another important limitation is that antipsychotic medication might have had an impact on the observed brain activity. Lastly, our task was primarily designed to investigate reality monitoring in schizophrenia and was not optimal for the study of visual processing. Our results, nonetheless, provide more evidence for the impact of affective symptoms on the functioning of schizophrenia patients. Anxiety and depression should be a target for treatment, as well as the object of further investigation, in this population since they seem to influence cognition even at a basic perceptual level. 


\section{Acknowledgements}

This work was supported by a Miguel Servet contract (CP09/00292) and grant PI10/02479 from the Instituto de Salud Carlos III - Subdirección General de Evaluación y Fomento de la Investigación Sanitaria - co-funded by Fondo Europeo de Desarrollo Regional (FEDER), both to GB; grant PRRMAB-A2011-19251 from the Sardinia Region to SS, and contract PTA2011-4983-I from the Ministerio de Ciencia e Innovación, Spain to CS-O. The study was also supported by the Centro de Investigación Biomédica en Red de Salud Mental (CIBERSAM), Instituto de Salud Carlos III. The authors thank Llobet Optics and Indo International S. A., who kindly donated the set of MRI-safe vision correction glasses used in this study.

Conflicts of interest: none 


\section{REFERENCES}

Ball, T.M., Stein, M.B., Ramsawh, H.J., Campbell-Sills, L., Paulus, M.P., 2014. Singlesubject anxiety treatment outcome prediction using functional neuroimaging. Neuropsychopharmacology 39, 1254-61. doi:10.1038/npp.2013.328

Bedwell, J.S., Rassovsky, Y., Orem, D.M., Kamath, V., 2011. The backward masking red light effect in schizophrenia: relationship to clinical features and neurocognitive performance. J. Abnorm. Psychol. 120, 490-6. doi:10.1037/a0022302

Brébion, G., Bressan, R.A., Pilowsky, L.S., David, A.S., 2009. Depression, avolition, and attention disorders in patients with schizophrenia: associations with verbal memory efficiency. J. Neuropsychiatry Clin. Neurosci. 21, 206-215.

Brébion, G., Gorman, J.M., Malaspina, D., Sharif, Z., Amador, X., 2001. Clinical and cognitive factors associated with verbal memory task performance in patients with schizophrenia. Am. J. Psychiatry 158, 758-64.

Brébion, G., Stephan-Otto, C., Huerta-Ramos, E., Ochoa, S., Usall, J., Abellán-Vega, H., Roca, M., Haro, J.M., 2015. Visual encoding impairment in patients with schizophrenia: contribution of reduced working memory span, decreased processing speed, and affective symptoms. Neuropsychology 29, 17-24. doi:10.1037/neu0000104

Brébion, G., Villalta-Gil, V., Autonell, J., Cervilla, J., Dolz, M., Foix, A., Haro, J.M., Usall, J., Vilaplana, M., Ochoa, S., 2013. Cognitive correlates of verbal memory and verbal fluency in schizophrenia, and differential effects of various clinical symptoms between male and female patients. Schizophr. Res. 147, 81-5. doi:10.1016/j.schres.2013.03.014

Brown, G.G., Ostrowitzki, S., Stein, M.B., von Kienlin, M., Liu, T.T., Simmons, A., Wierenga, C., Stein, O.Y., Bruns, A., Bischoff-Grethe, A., Paulus, M., 2015. Temporal profile of brain response to alprazolam in patients with generalized anxiety disorder. Psychiatry Res. 233, 394-401. doi:10.1016/j.pscychresns.2015.06.016

Cichy, R.M., Heinzle, J., Haynes, J.-D., 2012. Imagery and perception share cortical representations of content and location. Cereb. cortex 22, 372-80. doi:10.1093/cercor/bhr106

Clark, C.M., Chiu, C.G., Diaz, R.L., Goghari, V.M., 2014. Intact anger recognition in depression despite aberrant visual facial information usage. J. Affect. Disord. 165, 196-202. doi:10.1016/j.jad.2014.04.065

Gasquoine, P.G., 2014. Contributions of the insula to cognition and emotion.

Neuropsychol. Rev. 24, 77-87. doi:10.1007/s11065-014-9246-9 
Gomar, J.J., Ortiz-Gil, J., McKenna, P.J., Salvador, R., Sans-Sansa, B., Sarró, S., Guerrero, A., Pomarol-Clotet, E., 2011. Validation of the Word Accentuation Test (TAP) as a means of estimating premorbid IQ in Spanish speakers. Schizophr. Res. 128, 175-6. doi:10.1016/j.schres.2010.11.016

González-Hernández, J.A., Pita-Alcorta, C., Padrón, A., Finalé, A., Galán, L., Martínez, E., Díaz-Comas, L., Samper-González, J.A., Lencer, R., Marot, M., 2014. Basic visual dysfunction allows classification of patients with schizophrenia with exceptional accuracy. Schizophr. Res. 159, 226-33. doi:10.1016/j.schres.2014.07.052

Green, M.F., Lee, J., Cohen, M.S., Engel, S. a, Korb, A.S., Nuechterlein, K.H., Wynn, J.K., Glahn, D.C., 2009. Functional neuroanatomy of visual masking deficits in schizophrenia. Arch. Gen. Psychiatry 66, 1295-303. doi:10.1001/archgenpsychiatry.2009.132

Green, M.F., Lee, J., Wynn, J.K., Mathis, K.I., 2011. Visual masking in schizophrenia: overview and theoretical implications. Schizophr. Bull. 37, 700-8. doi:10.1093/schbul/sbr051

Grill-Spector, K., Kourtzi, Z., Kanwisher, N., 2001. The lateral occipital complex and its role in object recognition. Vision Res. 41, 1409-22.

Hartley, S., Barrowclough, C., Haddock, G., 2013. Anxiety and depression in psychosis: a systematic review of associations with positive psychotic symptoms. Acta Psychiatr. Scand. 128, 327-46. doi:10.1111/acps.12080

Harvey, P.-O., Lee, J., Cohen, M.S., Engel, S.A., Glahn, D.C., Nuechterlein, K.H., Wynn, J.K., Green, M.F., 2011. Altered dynamic coupling of lateral occipital complex during visual perception in schizophrenia. Neuroimage 55, 1219-26. doi:10.1016/j.neuroimage.2010.12.045

Lee, S.Y., Namkoong, K., Cho, H.H., Song, D.-H., An, S.K., 2010. Reduced visual P300 amplitudes in individuals at ultra-high risk for psychosis and first-episode schizophrenia. Neurosci. Lett. 486, 156-60. doi:10.1016/j.neulet.2010.09.035

Lysaker, P.H., Bell, M.D., Greig, T.C., Bryson, G.J., 2000. Emotional discomfort and impairments in verbal memory in schizophrenia. Psychiatry Res. 97, 51-9.

Lysaker, P.H., Salyers, M.P., 2007. Anxiety symptoms in schizophrenia spectrum disorders: associations with social function, positive and negative symptoms, hope and trauma history. Acta Psychiatr. Scand. 116, 290-8. doi:10.1111/j.16000447.2007.01067.x

Macevoy, S.P., 2013. "What?" and "where?" versus "what is where?": the impact of task on coding of object form and position in the lateral occipital complex. J. Vis. 13. doi:10.1167/13.8.21 
Makovac, E., Meeten, F., Watson, D.R., Garfinkel, S.N., Critchley, H.D., Ottaviani, C., 2016. Neurostructural abnormalities associated with axes of emotion dysregulation in generalized anxiety. NeuroImage Clin. 10, 172-181.

doi:10.1016/j.nicl.2015.11.022

Modi, S., Kumar, M., Kumar, P., Khushu, S., 2015. Aberrant functional connectivity of resting state networks associated with trait anxiety. Psychiatry Res. 234, 25-34. doi:10.1016/j.pscychresns.2015.07.006

Möser, C., Krieg, J.C., Zihl, J., Lautenbacher, S., 2006. Attention and memory deficits in schizophrenia: the role of symptoms of depression. Cogn. Behav. Neurol. 19, 150-6. doi:wnn.0000213910.41901.1a

Peng, D., Liddle, E.B., Iwabuchi, S.J., Zhang, C., Wu, Z., Liu, J., Jiang, K., Xu, L., Liddle, P.F., Palaniyappan, L., Fang, Y., 2015. Dissociated large-scale functional connectivity networks of the precuneus in medication-naïve first-episode depression. Psychiatry Res. Neuroimaging 232, 250-6. doi:10.1016/j.pscychresns.2015.03.003

Peralta, V., Cuesta, M.J., 1999. Dimensional structure of psychotic symptoms: an itemlevel analysis of SAPS and SANS symptoms in psychotic disorders. Schizophr. Res. 38, 13-26.

Perez, V.B., Shafer, K.M., Cadenhead, K.S., 2012. Visual information processing dysfunction across the developmental course of early psychosis. Psychol. Med. 42, 2167-79. doi:10.1017/S0033291712000426

Riskind, J.H., Kleiman, E.M., Seifritz, E., Neuhoff, J., 2014. Influence of anxiety, depression and looming cognitive style on auditory looming perception. J. Anxiety Disord. 28, 45-50. doi:10.1016/j.janxdis.2013.11.005

Rohlfing, T., Zahr, N.M., Sullivan, E. V, Pfefferbaum, A., Manuscript, A., 2010. The SRI24 multichannel atlas of normal adult human brain structure. Hum. Brain Mapp. 31, 798-819. doi:10.1002/hbm.20906

Sanfilipo, M., Lafargue, T., Rusinek, H., Arena, L., Loneragan, C., Lautin, A., Rotrosen, J., Wolkin, A., 2002. Cognitive performance in schizophrenia: relationship to regional brain volumes and psychiatric symptoms. Psychiatry Res. $116,1-23$.

Schennach-Wolff, R., Obermeier, M., Seemüller, F., Jäger, M., Messer, T., Laux, G., Pfeiffer, H., Naber, D., Schmidt, L.G., Gaebel, W., Klosterkötter, J., Heuser, I., Maier, W., Lemke, M.R., Rüther, E., Klingberg, S., Gastpar, M., Möller, H.-J., Riedel, M., 2011. Evaluating depressive symptoms and their impact on outcome in schizophrenia applying the Calgary Depression Scale. Acta Psychiatr. Scand. 123, 228-38. doi:10.1111/j.1600-0447.2010.01608.x 
Schultz, C.C., Wagner, G., Koch, K., Gaser, C., Roebel, M., Schachtzabel, C., Nenadic, I., Reichenbach, J.R., Sauer, H., Schlösser, R.G.M., 2013. The visual cortex in schizophrenia: alterations of gyrification rather than cortical thickness--a combined cortical shape analysis. Brain Struct. Funct. 218, 51-8. doi:10.1007/s00429-0110374-1

Sehatpour, P., Dias, E.C., Butler, P.D., Revheim, N., Guilfoyle, D.N., Foxe, J.J., Javitt, D.C., 2010. Impaired visual object processing across an occipital-frontalhippocampal brain network in schizophrenia: an integrated neuroimaging study. Arch. Gen. Psychiatry 67, 772-82. doi:10.1001/archgenpsychiatry.2010.85

Silverstein, S.M., Berten, S., Essex, B., Kovács, I., Susmaras, T., Little, D.M., 2009. An fMRI examination of visual integration in schizophrenia. J. Integr. Neurosci. 8, 175-202.

Slaghuis, W.L., Curran, C.E., 1999. Spatial frequency masking in positive- and negative-symptom schizophrenia. J. Abnorm. Psychol. 108, 42-50.

Tzourio-Mazoyer, N., Landeau, B., Papathanassiou, D., Crivello, F., Etard, O., Delcroix, N., Mazoyer, B., Joliot, M., 2002. Automated anatomical labeling of activations in SPM using a macroscopic anatomical parcellation of the MNI MRI single-subject brain. Neuroimage 15, 273-89. doi:10.1006/nimg.2001.0978

Vartanian, O., Skov, M., 2014. Neural correlates of viewing paintings: Evidence from a quantitative meta-analysis of functional magnetic resonance imaging data. Brain Cogn. 87C, 52-56. doi:10.1016/j.bandc.2014.03.004

Wei, X., Shen, H., Ren, J., Liu, W., Yang, R., Liu, J., Wu, H., Xu, X., Lai, L., Hu, J., Pan, X., Jiang, X., 2015. Alteration of spontaneous neuronal activity in young adults with non-clinical depressive symptoms. Psychiatry Res. 233, 36-42. doi:10.1016/j.pscychresns.2015.04.008

Wetherell, J.L., Palmer, B.W., Thorp, S.R., Patterson, T.L., Golshan, S., Jeste, D. V, 2003. Anxiety symptoms and quality of life in middle-aged and older outpatients with schizophrenia and schizoaffective disorder. J. Clin. Psychiatry 64, 1476-82.

Yin, Y., Hou, Z., Wang, X., Sui, Y., Yuan, Y., 2015. Association between altered resting-state cortico-cerebellar functional connectivity networks and $\mathrm{mood} / \operatorname{cognition}$ dysfunction in late-onset depression. J. Neural Transm. 122, 88796. doi:10.1007/s00702-014-1347-3 


\section{Figure 1. Schematic representation of the encoding phase of the fMRI task.}

The target items ( 45 words and 45 word/picture pairs) were presented one by one in pseudo-random order. Each stimulus slide was presented for 3.5 seconds. Fixation-crosses (duration between 2 to 5.5 seconds according to an exponential distribution) separated the stimuli. Participants were asked to indicate whether the presented items were vegetables or not, and to give their response by pressing one of two buttons ('vegetable' or 'other'). Responses were considered valid if they were provided during the presentation of the stimulus or the presentation of the subsequent fixation-cross. The total duration of the task was 10 minutes.

\section{Figure 2. Brain activity related to depression and anxiety during the viewing of pictures in the schizophrenia group.}

Top left: activation clusters positively associated with depression (red colour scale) at the bilateral Calcarine and right Precuneus for the contrast Pictures > Words. Top right: activation clusters negatively associated with anxiety (blue colour scale) and positively associated with depression (red colour scale) for the contrast Pictures $>$ Words. An activation cluster at the left Supramarginal Gyrus, common to both associations, shows purple colouring by addition of both colour scales. The blue-only cluster is located at the left Postcentral Gyrus. The $\mathrm{z}=-1$ and $\mathrm{z}=29$ planes of the SRI24 structural template are shown for illustration purposes only, following the neurological convention (Table 2 presents all significant activations in detail). Bottom: partial regression plots illustrating the opposite associations of mean cluster response at the left Supramarginal Gyrus with depression, controlled for anxiety (left) and with anxiety, controlled for depression (right). 
Table 1. Activations observed for the Pictures > Words contrast for each group. In the 26 healthy participants, verbal IQ was controlled for, and a cluster-defining voxel-level $p_{F W E}<0.05$ was used. In the 20 schizophrenia patients, a cluster-defining voxel-level uncorrected $\mathrm{p}<.001$ was used followed by a cluster extent threshold corresponding to $\mathrm{p}_{\mathrm{FWE}}<0.05$; results are shown before and after controlling for illness duration.

\begin{tabular}{|c|c|c|c|}
\hline \multirow{2}{*}{$\begin{array}{l}\text { Cluster size and FWE- } \\
\text { corrected cluster-level } \\
\text { significance }\end{array}$} & \multicolumn{2}{|c|}{ Cluster peak coordinates * } & \multirow[t]{2}{*}{ Region } \\
\hline & SRI24 & MNI & \\
\hline \multicolumn{4}{|c|}{ HEALTHY PARTICIPANTS } \\
\hline \multirow[t]{2}{*}{$724(0.001)$} & & & \\
\hline & & $33,-84,19$ & Middle occipital gyrus R \\
\hline \multirow[t]{2}{*}{$547(0.001)$} & $-27,-67,-16$ & $-30,-70,-7$ & Fusiform gyrus L + \\
\hline & $-3,-85,-19$ & $-4,-89,6$ & Lingual R/L \\
\hline $102(0.001)$ & $-33,-82,-4$ & $-37,-82,11$ & Middle occipital gyrus L \\
\hline \multicolumn{4}{|c|}{ SCHIZOPHRENIA PATIENTS } \\
\hline \multirow[t]{3}{*}{$913(0.001)$} & $-3,-85,-16$ & $-4,-88,-2$ & Lingual R/L + \\
\hline & $21,-76,-19$ & $22,-79,-8$ & Fusiform gyrus R + \\
\hline & $-33,-49,-19$ & $-36,-52,-15$ & Fusiform gyrus L \\
\hline $49\left(0.071^{\mathrm{a}}\right)$ & $27,-82,8$ & $29,-80,25$ & Middle occipital gyrus $\mathrm{R}$ \\
\hline \multicolumn{4}{|l|}{$\begin{array}{l}\text { Controlling for illness } \\
\text { duration }\end{array}$} \\
\hline \multirow[t]{3}{*}{$993(0.001)$} & $3,-85,-16$ & $-4,-88,-2$ & Lingual $\mathrm{R} / \mathrm{L}+$ \\
\hline & $21,-76,-19$ & $22,-79,-8$ & Fusiform gyrus R + \\
\hline & $-33,-46,-19$ & $-36,-49,-15$ & Fusiform gyrus L \\
\hline $62(0.027)$ & $27,-82,8$ & $29,-80,25$ & Middle occipital gyrus $\mathrm{R}$ \\
\hline $55(0.042)$ & $-24,-85,8$ & $-27,-83,25$ & Middle occipital gyrus L \\
\hline
\end{tabular}

* Bold coordinates denote a peak-level corrected significance $\mathrm{p}_{\mathrm{FWE}}<.05$.

a Activation not significant in terms of its cluster extent. 
Table 2. Brain activations associated with depression and anxiety during the presentation of pictures (contrast $P>W$ ) in the 20 schizophrenia patients. Depression and anxiety were entered together in the analysis.

\begin{tabular}{|c|c|c|c|}
\hline \multirow{2}{*}{$\begin{array}{l}\text { Cluster size and cluster- } \\
\text { level significance (pFwE) }\end{array}$} & \multicolumn{2}{|c|}{ Cluster peak coordinates* } & \multirow[t]{2}{*}{ Region } \\
\hline & SRI24 & MNI & \\
\hline $\begin{array}{l}\text { Depression } \\
\text { (positive association) }\end{array}$ & & & \\
\hline $121(0.001)$ & $-6,-85,-4$ & $-7,-85,12$ & Calcarine $\mathrm{R} / \mathrm{L}$ \\
\hline $72(0.012)$ & $18,-52,-1$ & $19,-51,7$ & Precuneus $\mathrm{R}$ \\
\hline $50(0.048)$ & $-39,-43,29$ & $-43,-35,39$ & Supramarginal gyrus L \\
\hline $\begin{array}{l}\text { Anxiety } \\
\text { (negative association) }\end{array}$ & & & \\
\hline $63(0.027)$ & $-60,-13,29$ & $-65,-5,32$ & Postcentral gyrus L \\
\hline $49\left(0.063^{\mathrm{a}}\right)$ & $-39,-43,29$ & $-43,-35,39$ & Supramarginal gyrus L \\
\hline
\end{tabular}

Note: an initial voxel-level significance threshold $p<.001$ was used.

* Bold coordinates denote a peak-level corrected significance $\mathrm{p}_{\mathrm{FWE}}<.05$.

${ }^{a}$ Activation not significant in terms of its cluster extent. 
Table 3. Brain activations associated with illness duration during the presentation of pictures (contrast Pictures $>$ Words) in the 20 schizophrenia patients (all associations are negative).

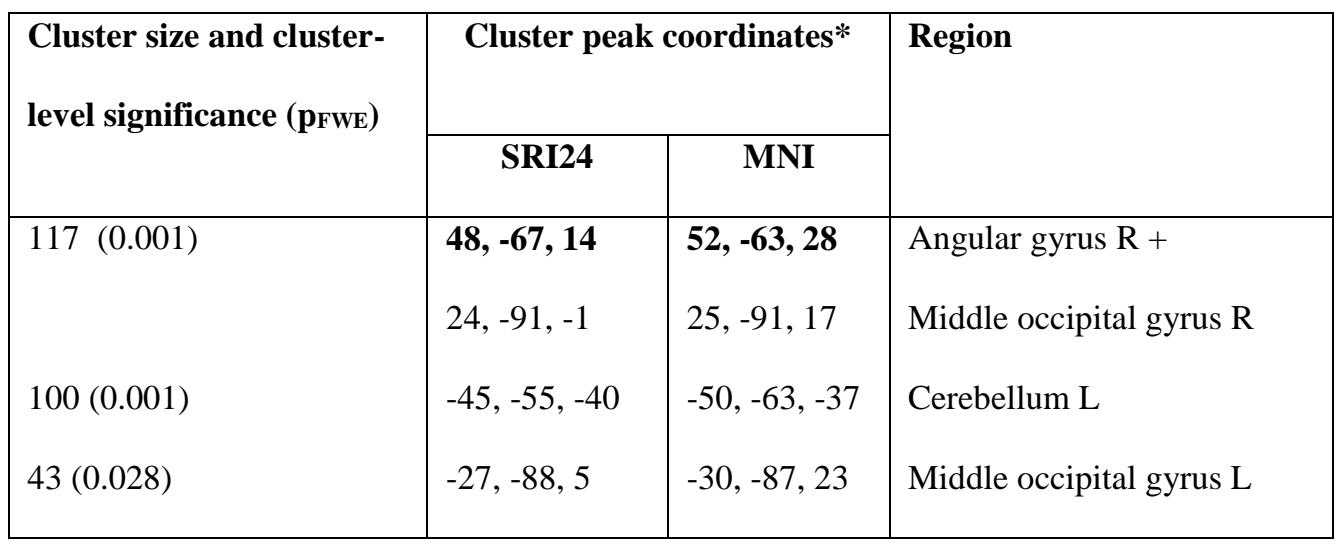

Note: an initial voxel-level significance threshold $\mathrm{p}<.001$ was used.

* Bold coordinates denote a peak-level corrected significance $\mathrm{p}_{\mathrm{FWE}}<.05$. 
Figure 1

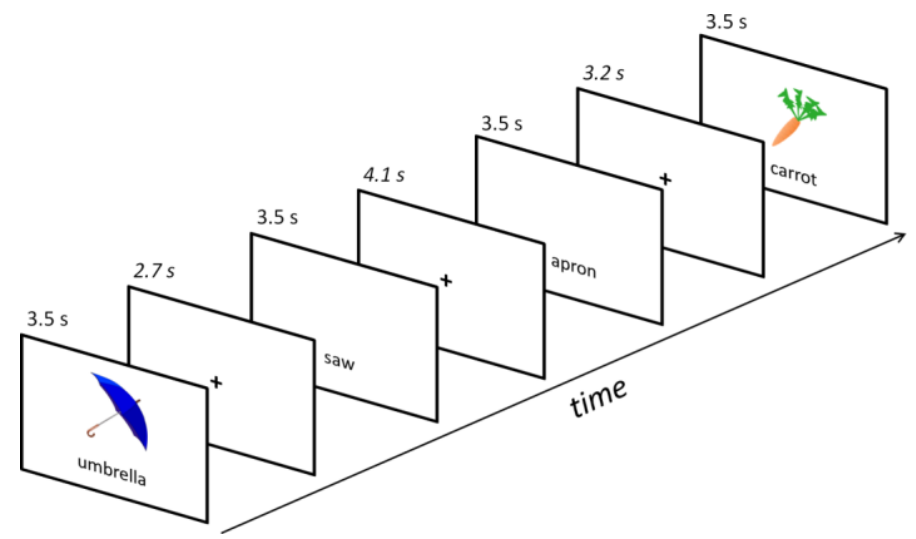


Figure 2

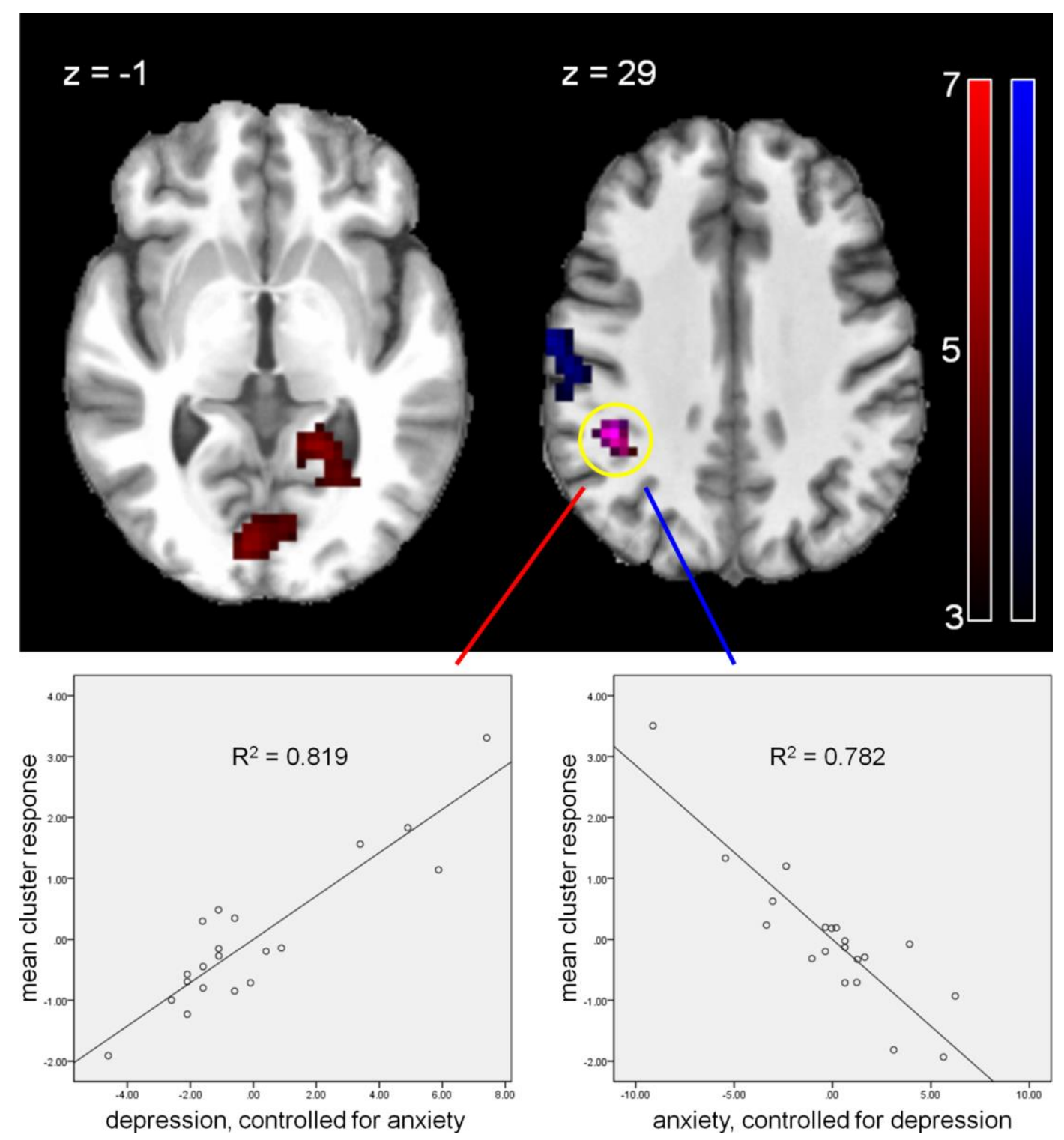

